\title{
Compositional MRI of the anterior cruciate ligament of professional alpine ski racers: preliminary report on seasonal changes and load sensitivity
}

\author{
Robert Csapo ${ }^{1}$, Vladimir Juras ${ }^{2^{*}}$ (D), Bernhard Heinzle ${ }^{3}$, Siegfried Trattnig ${ }^{2,4}$ and Christian Fink ${ }^{1,5}$
}

\begin{abstract}
The purpose of this study was to investigate potential changes in the anterior cruciate ligament $(A C L)$ structure of alpine ski racers over the course of an entire season using quantitative magnetic resonance imaging (T2* mapping). The dominant legs of three alpine ski racers were examined on a 3-T MR scanner four times at 3-month intervals. Multi-echo sequences for T2* maps, which were coregistered with high-resolution morphological sequences for reproducible definition of $\mathrm{ACL}$ regions of interest, were acquired. Means and standard deviations of $T 2^{*}$ values from the central and femoral portion of the ACL were extracted and presented in a descriptive manner. $T 2^{*}$ values were subject to seasonal changes, which were most pronounced in the ligament central region. Substantial increases (+ $41 \%)$ occurred between the measurements taken in January and April. A partial recovery of T2* (-19\%) was observed in the July follow-up. The increased T2* times may reflect decreased stress tolerance and increased susceptibility for fatigue tears at the end of the competitive season. Further research in larger samples is required. The likeliness of ACL tears may depend on the precedent history of mechanical loading and vary in professional athletes over the course of the competitive season.
\end{abstract}

Keywords: Athletes, Anterior cruciate ligament, Magnetic resonance imaging, Skiing, Seasons

\section{Key points}

- Quantitative magnetic resonance imaging can detect seasonal changes in cruciate ligaments of ski racers.

- Anterior cruciate ligament (ACL) T2* values peaked in April at the end of season.

- ACL T2* increase may reflect a decreased stress tolerance increasing the risk of fatigue tears.

\section{Background}

Tears of the anterior cruciate ligament (ACL) represent one of the most frequent musculoskeletal injuries and

\footnotetext{
*Correspondence: vladimir.juras@meduniwien.ac.at

${ }^{2}$ Highfield MR Center, Department of Biomedical Imaging and Image-Guided Therapy, Medical University of Vienna, Lazarettgasse 14, A-1090 Vienna,

Austria

Full list of author information is available at the end of the article
}

typically affect young and athletic populations $[1,2]$. In alpine ski racing, a discipline characterised by high speeds and large forces imposed on the lower limbs, ACL tears are particularly common, with epidemiological data from the International Ski Federation Injury Surveillance System indicating an incidence of 5.4-8.5 injuries per 100 skier-seasons [3, 4]. While the typical mechanisms of ACL injury in alpine ski racing, namely the dynamic snow plow, slip catch, and back-weighted landing [5], are well described, observations of ACL tears occurring consequent to apparently minor stresses (and often not even resulting in crashes) raise questions about possible intrinsic factors predisposing athletes to injury.

Yet another parameter potentially associated with ACL injury risk is the precedent history of ACL loading. In vitro studies using cadaveric limbs have shown that 
repeated exposure to submaximal knee loading may alter the ACL stress tolerance, leading to sudden tear $[6,7]$. Anecdotal evidence derived from the database of our sports clinic further suggests that the number of ACL reconstructions performed in professional alpine ski racers accumulates in the later stages of the competitive season. While this observation may be caused by a number of factors unrelated to the ACL mechanical properties, such as changes in environmental and snow conditions and neuromuscular or cognitive fatigue, alterations in the ligament's microstructural and biochemical properties promoting fatigue tears cannot be ruled out.

One approach to obtaining imaging-based biomarkers indicative of the ACL structure and biochemistry is through magnetic resonance imaging (MRI)-based $\mathrm{T} 2$ * mapping. As is well-known, T2* values reflect the decay of transverse magnetisation caused by spin-spin relaxation and magnetic field inhomogeneities:

$$
\frac{1}{T 2^{*}}=\frac{1}{T 2}+\gamma \Delta B
$$

where T2 is the "true" transverse relaxation time, $\gamma$ is the gyromagnetic ratio, and $\Delta \mathrm{B}$ reflects the magnetic field inhomogeneities across a voxel [8]. T2\% values reflect inherent tissue properties that are related to tissue composition (e.g., collagen fibre and water content) and organisation. In collagenous tissues, shorter $\mathrm{T} 2 \%$ relaxation times have been found to be positively correlated to a more highly organised (i.e., healthier) structure of menisci [9] and ligaments [10], whereas in cartilage $\mathrm{T} 2$ * values are reportedly lower in degraded as compared to healthy tissue [11]. Thus, T2* maps may provide regionspecific information about the integrity of different collagenous tissues, including the ACL [12].

The purpose of the $\mathrm{T}^{*}$ measurements in addition to conventional morphological MRI reported in this manuscript was to investigate potential changes in the ACL structure of alpine ski racers over the course of an entire season.

\section{Methods}

Four male professional alpine skiers engaged at the European and World Cup level volunteered to participate in the study. One athlete suffered an ACL tear after the first measurement, so the study could be completed in three subjects, aged 23.3, 21.3, and 21.4 years, weighing 86,80 , and $76 \mathrm{~kg}$, with a height of 182,180 , and 180 $\mathrm{cm}$, respectively. All subjects participated exclusively in technical events (slalom and giant slalom). The participants were scheduled for four visits evenly distributed over the 2018-2019 season (appointments in October 2018, January 2019, April 2019, and July 2019). The study was approved by the ethics committee of the
Medical University of Innsbruck (vote AN2016-0172), and all participants gave written informed consent.

\section{Imaging protocol}

Images were acquired at a private hospital on a 3-T whole-body scanner (Skyra, Siemens Healthineers, Erlangen, Germany) using a 6-channel flex coil. Subjects were positioned supine, with the knee of the dominant leg, defined as the leg preferentially used to kick a ball, fixed in an MRI-safe pneumatic knee fixation device at $10^{\circ}$ of articular flexion (Fig. 1).

The MRI protocol consisted of the following consecutive sequences:

1) A high-resolution, fat-saturated proton density (PD)-weighted, turbo spin echo (TSE) sequence along a parasagittal plane;

2) A high-resolution isotropic, fat-saturated, isotropic PD-weighted sampling perfection with application of optimised contrasts using different flip angle evolution (PD SPACE) sequence, acquired at rest along a parasagittal plane;

3) A multi-echo T2*-weighted gradient-echo (GRE) sequence to measure $\mathrm{T} 2 *$ relaxation values;

4) A high-resolution isotropic, fat-saturated, isotropic PD SPACE sequence, as described at point 2;

5) A multi-echo T2*-weighted GRE sequence, as described at point 3 .

Details regarding technical parameters of these MRI sequence are reported in Table 1.

First, sequences 1,2 , and 3 were acquired at rest. Then, the pneumatic system within the knee fixation device was used to apply 2.5 bar of pressure, equivalent to a force of $150 \mathrm{~N}$, against the posterior aspect of the calf in order to provoke a Lachman test-like scenario, pushing the tibia against the fixed femur in the anterior direction and, thus, straining the ACL. During ongoing pressure application, sequences 4 and 5 were completed.

\section{Image postprocessing and evaluation}

Using the multi-echo images obtained with sequences 3 and 5 , the $\mathrm{T} 2 *$ relaxation times were estimated by fitting a monoexponential decay function to the signal intensities (SI) of voxels that decrease with increasing time of echo (TE):

$$
S I(T E)=S_{0} e^{-\frac{T E}{T_{2}^{*}}}
$$

To analyse the resultant $\mathrm{T} 2 *$ values, regions of interest (ROIs) were placed over the central and femoral region of the ACL. The tibial third of the ACL was deliberately omitted as measurements obtained in this region were considered to be most prone to bias related to focally 


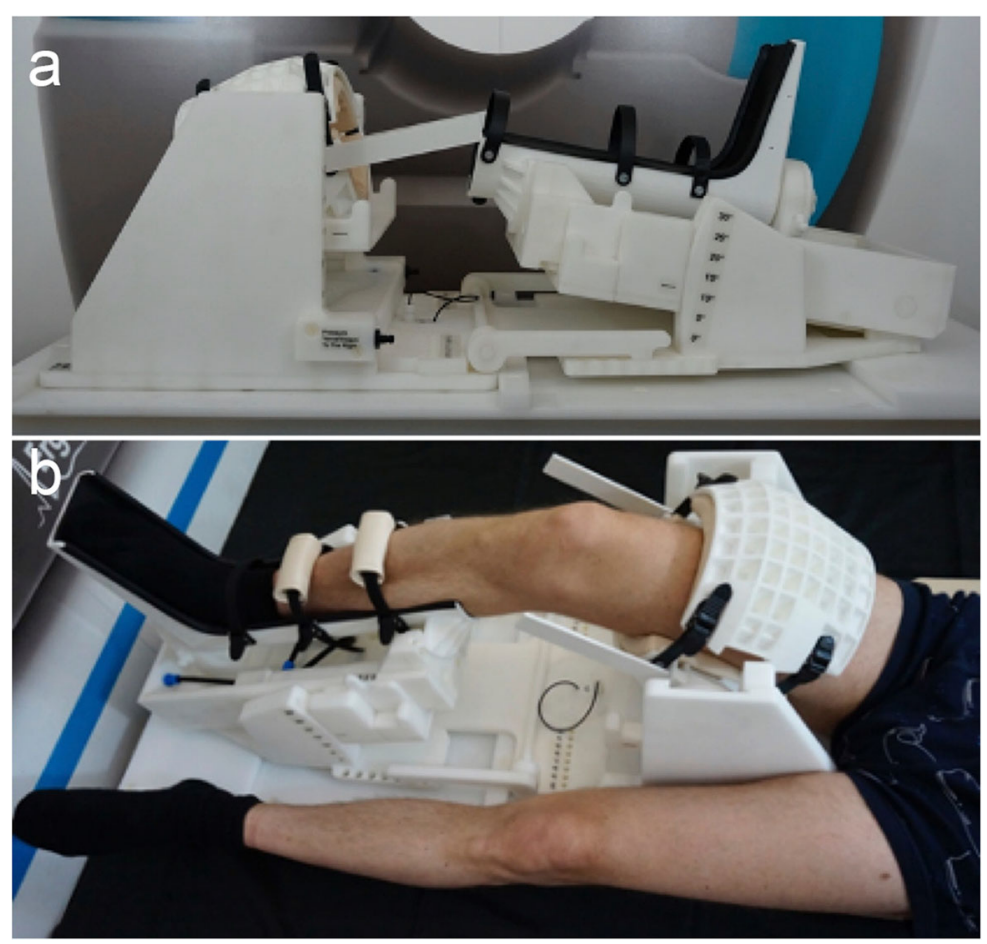

Fig. 1 a MRI-safe, pneumatically driven knee fixation device. The "boot" of the device may be rotated upwards, to exert pressure against the posterior aspect of the calf, with the thigh being firmly anchored. This creates a Lachman test-like scenario in which the anterior cruciate ligament is strained. $\mathbf{b}$ Subject positioning within the device

increased signal intensities, which were physiologically implausible and presumably due to field orientation (also known as magic angle) artefacts [13]. To warrant consistent in-plane ROI positioning across pressure conditions and measurement points, automated image coregistration was employed. For this purpose, the highresolution three-dimensional TSE sequence was aligned to T2* maps by cubic interpolation, making use of the image orientation information provided in the DICOM header. Then, corresponding high-resolution slices were selected in order to match with T2* maps.

As only axial displacement and shear was expected, multimodal affine transformation was used to register $\mathrm{T} 2 *$ maps onto a corresponding high-resolution slice using an optimiser function (initial radius $=0.001$; epsilon $=1.5 \mathrm{e}^{-4}$; growth factor $=1.01 ;$ maximum iterations $=$ 300). All further maps were coregistered to the $\mathrm{T} 2 *$ map obtained during the first visit without load. The ROIs were placed onto a first-visit high-resolution slice and automatically transferred onto the other $\mathrm{T} 2 *$ maps. All automatically generated ROIs were visually inspected for artefacts characterised by uncommonly high $\mathrm{T} 2 *$ values (red spots on T2* maps). The corresponding voxels were manually excluded from further analysis. Figure 2 shows demonstrative images of the high-resolution threedimensional TSE sequence with ACL ROIs, the corresponding $\mathrm{T}^{*}$ map, and a T2*-weighted image with superimposed colour-coded $\mathrm{T} 2 *$ value overlay. Using

Table 1 The MR sequence parameters

\begin{tabular}{llll}
\hline & Proton density-weighted & Proton density-weighted & T2*-weighted \\
\hline Type & Turbo spin-echo & SPACE & Gradient echo \\
Fat saturation & Yes & Yes & No \\
Time of repetition (ms) & 2,230 & 700 & 575 \\
Time of echo (ms) & 33 & 30 & $3.71,10.56,17.99,25.42,32.85,40.28$ \\
Echo train length & 7 & 23 & - \\
Bandwidth (Hz/pixel) & 250 & 385 & 260 \\
In-plane resolution $(\mathrm{mm} \times \mathrm{mm})$ & $0.42 \times 0.42$ & $0.66 \times 0.66$ & $0.56 \times 0.56$ \\
Through-plane resolution $(\mathrm{mm})$ & 2 & 0.70 & 2 \\
\hline
\end{tabular}




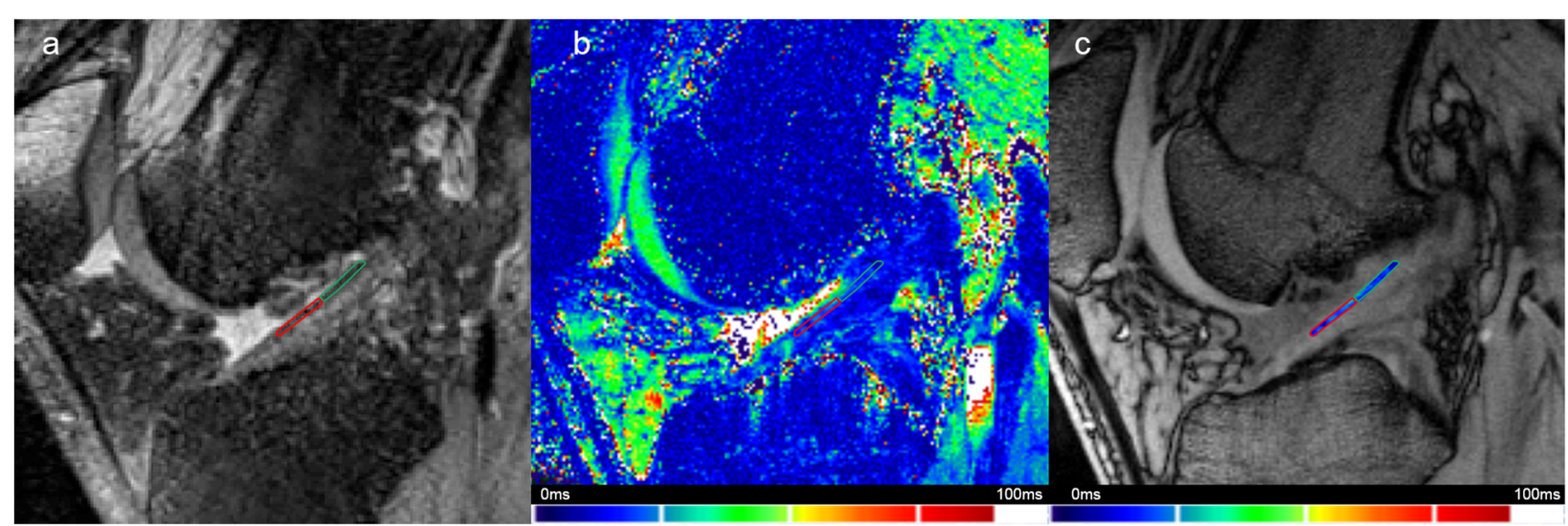

Fig. 2 Demonstrative images showing a T2* map with regions of interest located into the anterior cruciate ligament (a), the corresponding colour-coded T2* map (b), and a T2*-weighted image with superimposed colour-coded T2* value overlay (c)

descriptive statistics, the means and standard deviations of $\mathrm{T}_{2}^{*}$ values were separately extracted for the central and femoral region. All image post-processing was performed using custom-made scripts written in MATLAB (64-bit version R2019a, The MathWorks Inc., Natick, MA, USA).

Due to the small sample $(n=3)$, individual and mean data are presented.

\section{Results}

Individual $\mathrm{T}^{*}$ relaxation times by $\mathrm{ACL}$ region, pressure condition, and phase of the season are shown in Table 2.

On average, baseline measurements of $\mathrm{T} 2 \%$ at rest showed values of (mean value) 11.1 and $9.5 \mathrm{~ms}$ in the central and femoral ACL portion, respectively. While T2* values remained fairly stable between the measurements obtained in October and January, increases of 40.7\% (central ACL portion) and 22.4\% (femoral ACL portion) were observed between January and April. During the off-season (April-July), measures obtained in the central ACL region returned to baseline while they remained at an elevated level in the femoral portion.

During the application of pressure, $\mathrm{T} 2$ * baseline mean values were 12.9 and $11.4 \mathrm{~ms}$ in the central and femoral ACL region, respectively. Just as in the measurements obtained at rest, $\mathrm{T}^{*}$ values in the central $\mathrm{ACL}$ region increased during the observation period, with the most prominent rises occurring between January and April (+ $24.6 \%$ ). In the femoral region, by contrast, no substantial changes were observed in the same period (-4.6\%). Figure 3 shows the seasonal changes in average $\mathrm{T} 2 \%$ values as measured in the central ACL ROI at rest and during pressure application.

\section{Discussion}

The development of noninvasive in vivo imaging-based biomarkers indicative of the ACL microstructure is of utmost clinical interest, as this might allow for conclusions about its mechanical properties and stress tolerance to be drawn. Such a tool would also be applicable to the assessment of the healing process following ACL

Table 2 Individual T2* values (ms) by anterior cruciate ligament region, pressure condition, and phase of the season

\begin{tabular}{|c|c|c|c|c|c|c|c|c|}
\hline & \multicolumn{2}{|l|}{ October } & \multicolumn{2}{|l|}{ January } & \multicolumn{2}{|l|}{ April } & \multicolumn{2}{|l|}{ July } \\
\hline & Central & Femoral & Central & Femoral & Central & Femoral & Central & Femoral \\
\hline \multicolumn{9}{|c|}{ Measurements obtained at rest } \\
\hline Subject 1 & $9.0 \pm 3.4$ & $8.2 \pm 2.0$ & $9.6 \pm 2.6$ & $8.0 \pm 1.8$ & $15.2 \pm 3.4$ & $13.9 \pm 5.7$ & $12.0 \pm 3.1$ & $11.5 \pm 5.6$ \\
\hline Subject 2 & $10.3 \pm 3.3$ & $10.3 \pm 3.1$ & $11.2 \pm 2.3$ & $12.7 \pm 7.1$ & $16.9 \pm 4.1$ & $13.1 \pm 8.4$ & $12.8 \pm 2.9$ & $15.5 \pm 4.2$ \\
\hline Subject 3 & $13.9 \pm 2.6$ & $10.1 \pm 2.0$ & $8.4 \pm 2.7$ & $8.6 \pm 2.7$ & $8.9 \pm 2.2$ & $8.9 \pm 1.9$ & $8.4 \pm 2.4$ & $10.3 \pm 1.9$ \\
\hline \multicolumn{9}{|c|}{ Measurements obtained during pressure application } \\
\hline Subject 1 & $11.0 \pm 2.6$ & $8.9 \pm 1.4$ & $10.9 \pm 2.3$ & $9.3 \pm 2.2$ & $15.2 \pm 3.1$ & $11.2 \pm 3.8$ & $15.6 \pm 3.0$ & $12.3 \pm 5.1$ \\
\hline Subject 2 & $14.5 \pm 3.6$ & $11.3 \pm 3.0$ & $14.5 \pm 1.8$ & $16.4 \pm 4.3$ & $20.9 \pm 6.7$ & $13.5 \pm 4.1$ & $19.6 \pm 4.9$ & $15.7 \pm 5.2$ \\
\hline Subject 3 & $13.2 \pm 3.5$ & $14.0 \pm 2.1$ & $15.1 \pm 2.5$ & $10.1 \pm 2.6$ & $14.5 \pm 2.2$ & $9.4 \pm 1.9$ & $8.8 \pm 2.1$ & $9.6 \pm 1.6$ \\
\hline
\end{tabular}




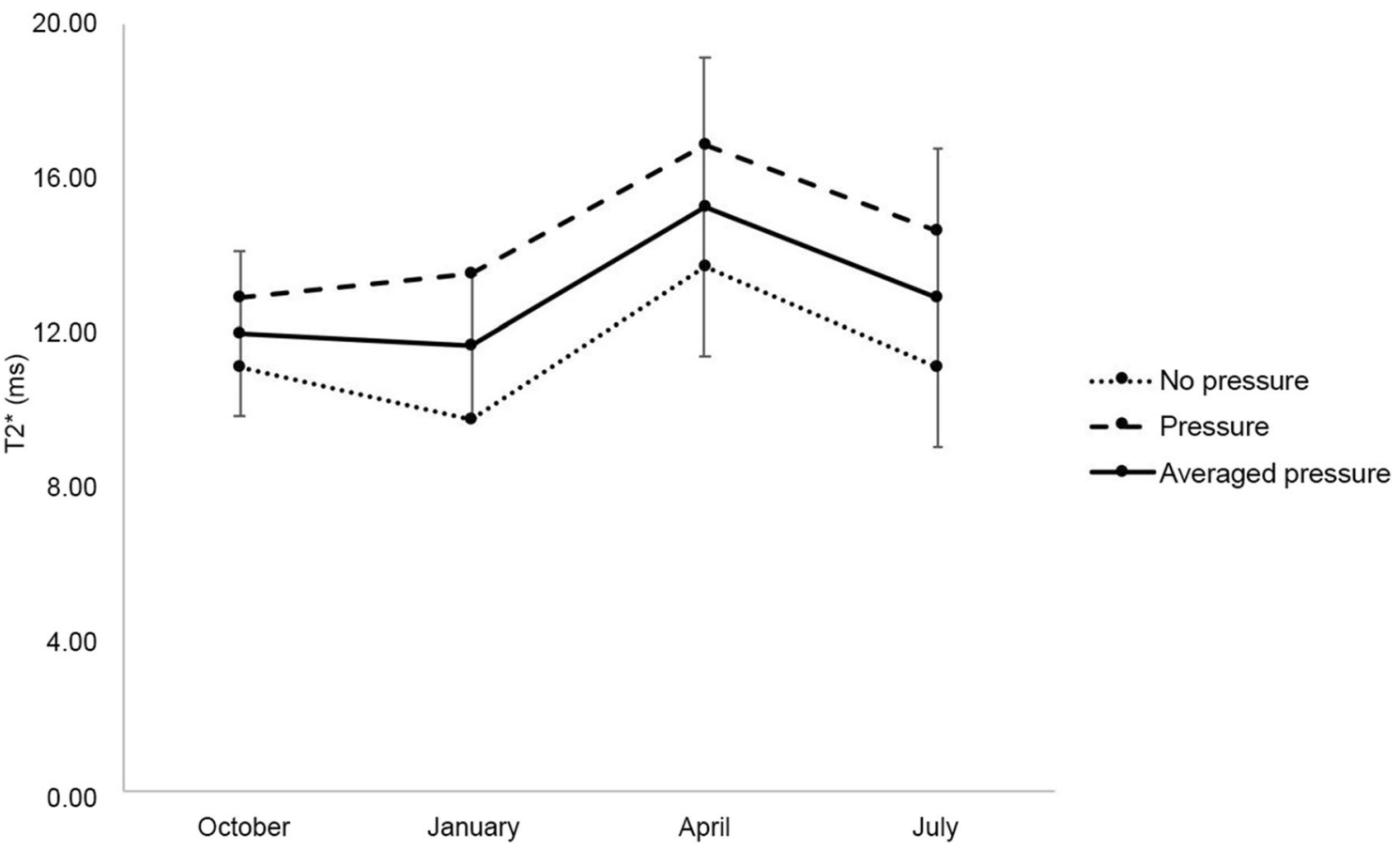

Fig. $3 \mathrm{TZ}^{*}$ values as measured in the central anterior cruciate ligament region averaged across subjects. Error bars are only shown for the average of measures obtained at rest and during pressure application (solid line in the middle)

reconstruction. One possible approach to obtaining such biomarkers that has received increased attention in the past decade relies on the MRI-based T2* mapping technique $[12,14-17]$. T2* relaxation times are characteristic values that provide quantitative information about the tissue structure and composition, in particular the collagen fibre content and organisation, on a voxel-by-voxel basis. Aiming to provide evidence indicative of a weakening of the ACL structure that might promote fatigue tears and explain a potential accumulation of injuries in the later stages of the competitive season, we applied the $\mathrm{T} 2$ * mapping technique to longitudinally study three professional alpine ski racers over the course of an entire season. The main finding of our pilot experiments is that $\mathrm{T}^{*}$ values as measured under different loading conditions peak in April, corresponding with the end of the competitive season in alpine ski racing.

In one of the first studies proposing $\mathrm{T} 2 \%$ for the evaluation of the structure (and tensile properties) of the ACL, Biercevicz et al. [16] used a porcine model and found the volume and median greyscale in $\mathrm{T} 2 \%$-weighted images to predict the maximum failure load of patellar tendon allografts used for ACL reconstruction. Since signal intensities are sequence- and magnet-dependent [18], the same group then proceeded to refine the technique and use $\mathrm{T} 2$ * relaxation times, which were found to correlate well with the results of tensile failure tests and the histologically assessed ligament maturity index of repaired minipig
ACLs $[14,15]$. According to these studies, lower T2* values coincide with greater stress tolerance and ligament maturity. Validation in human cadavers has proven to be more challenging, which may be due to the restricted variability of $\mathrm{T}_{2}^{*}$ relaxation times measured in cadaveric ligaments [17]. The joint body of evidence published to date, however, suggests that $\mathrm{T}^{*}$ mapping is a promising tool for the in vivo evaluation of ACL quality. This notion is also supported by a recent report testifying to excellent intra-rater (intraclass correlation coefficient 0.98) and inter-rater reliability (intraclass correlation coefficient 0.90) of T2* values measured in the ACL [19].

At rest, the $\mathrm{T}^{*}$ * times measured in our study ranged between 8.4 and $16.9 \mathrm{~ms}$ in the central and between 8.0 and $15.5 \mathrm{~ms}$ in the femoral ACL region. These values agree well with those reported by Anz et al. [19] but are slightly lower than those found by Schmitz et al. [12], who reported ranges of 15.9-25.1 $\mathrm{ms}$ and 13.9-30.6 ms, respectively, in recreationally active men of similar age. Differences in the image acquisition protocol may explain the discrepant findings. Since Schmitz et al. [12] obtained the first echo only after $8.3 \mathrm{~ms}$ (the shortest TE used in our study was $3.7 \mathrm{~ms}$ ), the shape of their signal intensity decay curve may have appeared with a different shape, thus yielding longer $\mathrm{T} 2 *$ times. Of note, our preliminary study was the first to measure $\mathrm{T}^{*}$ in the strained ACL. During the application of pressure, T2* values 
were greater by up to $33 \%$ and $20 \%$ in the central and femoral region of the ACL, respectively.

Stress MRI examinations have been performed in knee articular cartilage [20,21]. These studies showed small, yet statistically significant load-associated decreases in T2 relaxation times, which were explained by cartilage compression and extrusion of water, leading to a more compressed collagen and proteoglycan structure. The stress tests performed in our study, by contrast, acted to extend the ACL. Thus, it is plausible to assume that the density of collagens and other solid ACL components was lower during pressure application, resulting in the observed increase in $\mathrm{T} 2 *$ values.

The primary findings of this study are the seasonal changes of $\mathrm{T}_{2} *$ relaxation times in our sample of professional alpine ski racers that were most pronounced in the central region of the ACL and could be observed both at rest and during application of pressure. While average $\mathrm{T} 2 *$ values remained fairly constant between the measurements obtained in October and January, there was a marked increase of $41 \%$ (resting measurements) between January and April. Longer ACL T2* relaxation times have been associated with lower cell density and collagen organisation [15] and tensile load to failure [14]. Acknowledging the small sample size and explorative nature of the study, our data are, therefore, indicative of decreased ACL stress tolerance capacity in the end phase of the competitive season. The subsequent decrease of $\mathrm{T}^{*}$ values between April and July (-19\%) might be interpreted as a partial recovery of the ACL structure. Further studies involving larger samples are required to substantiate these hypotheses.

This pilot study is subject to a number of limitations. First and foremost, the small sample size $(n=3)$ precluded us from performing inferential statistics, which is why our results must be considered preliminary. However, it should be pointed out that a substantial increase in T2* values between January and April was consistently observed in all three subjects. In addition, implausibly bright spots presumably resulting from magic angle artefacts [13] precluded the analysis of $\mathrm{T} 2 \%$ values in the ACL proximal third. Furthermore, no direct measures of the ACL mechanical properties could be obtained, so the postulated correlation of $\mathrm{T} 2 \%$ values with the ligament stress tolerance relies solely on findings from earlier studies in animals $[14,15]$. Future studies might consider including stress imaging. Finally, it must be mentioned that, although $\mathrm{T} 2 *$ maps were coregistered with high-resolution images to warrant consistent inplane ROI placement, minor differences due to not perfectly matched slices cannot be ruled out.

In conclusion, the results of this preliminary study show seasonal changes of $\mathrm{T} 2 \%$ values mapped in the ACL of professional alpine ski racers, which are most pronounced in the ligament's central region. The values were found to peak in April, which coincides with the end of the competitive season and may reflect a decrease in stress tolerance, potentially increasing the risk of fatigue tears. Further research in larger samples is required.

\section{Abbreviations \\ ACL: Anterior cruciate ligament; GRE: Gradient echo; MRI: Magnetic resonance imaging; PD: Proton density; ROI: Region of interest; \\ SPACE: Sampling perfection with application optimised contrasts using different flip angle evolutions; TE: Time of echo; TSE: Turbo spin echo}

\section{Authors' contributions}

All authors should have made substantial contributions to all of the following: (1) the conception and design of the study, or acquisition of data, or analysis and interpretation of data, (2) drafting the article or revising it critically for important intellectual content, (3) final approval of the version to be submitted.

\section{Funding}

This pilot study has been financially supported by a research grant of the Austrian Skiing Federation.

Availability of data and materials

Data are available based on reasonable request from corresponding author.

\section{Ethics approval and consent to participate}

The study was approved by the ethics committee of the Medical University of Innsbruck (vote AN2016-0172), and all participants gave written informed consent.

\section{Consent for publication}

Included in the written informed consent

\section{Competing interests}

The authors declare that they have no competing interests.

\section{Author details}

${ }^{1}$ Research Unit for Orthopaedic Sports Medicine and Injury Prevention, ISAG, University for Health Sciences, Medical Informatics and Technology, Hall A-6060, Austria. ${ }^{2}$ Highfield MR Center, Department of Biomedical Imaging and Image-Guided Therapy, Medical University of Vienna, Lazarettgasse 14, A-1090 Vienna, Austria. ${ }^{3}$ Kursana Private Hospital, Wörgl A-6030, Austria. ${ }^{4}$ CD Laboratory for Molecular Clinical MR Imaging, Vienna, Austria. ${ }^{5}$ Gelenkpunkt Sports and Joint Surgery, Innsbruck A-6020, Austria.

Received: 19 August 2020 Accepted: 28 October 2020

Published online: 23 November 2020

\section{References}

1. Beynnon BD, Vacek PM, Newell MK et al (2014) The effects of level of competition, sport, and sex on the incidence of first-time noncontact anterior cruciate ligament injury. Am J Sports Med 42:1806-1812. https:// doi.org/10.1177/0363546514540862

2. Prodromos CC, Han Y, Rogowski J, Joyce B, Shi K (2007) A meta-analysis of the incidence of anterior cruciate ligament tears as a function of gender, sport, and a knee injury-reduction regimen. Arthroscopy 23:1320-1325.e6. https://doi.org/10.1016/j.arthro.2007.07.003

3. Pujol N, Rousseaux Blanchi MP, Chambat P (2007) The incidence of anterior cruciate ligament injuries among competitive alpine skiers: a 25-year investigation. Am J Sports Med 35:1070-1074. https://doi.org/10.1177/ 0363546507301083

4. Bere T, Flørenes TW, Nordsletten L, Bahr R (2014) Sex differences in the risk of injury in World Cup alpine skiers: a 6-year cohort study. Br J Sports Med 48:36-40. https://doi.org/10.1136/bjsports-2013-092206

5. Bere T, Flørenes TW, Krosshaug T et al (2011) Mechanisms of anterior cruciate ligament injury in world cup alpine skiing: a systematic video analysis of 20 cases. Am J Sports Med 39:1421-1429. https://doi.org/10. $1177 / 0363546511405147$ 
6. Lipps DB, Wojtys EM, Ashton-Miller JA (2013) Anterior cruciate ligament fatigue failures in knees subjected to repeated simulated pivot landings. Am J Sports Med 41:1058-1066. https://doi.org/10.1177/0363546513477836

7. Wojtys EM, Beaulieu ML, Ashton-Miller JA (2016) New perspectives on ACL injury: on the role of repetitive sub-maximal knee loading in causing ACL fatique failure. J Orthop Res 34:2059-2068. https://doi.org/10.1002/jor.23441

8. Chavhan GB, Babyn PS, Thomas B, Shroff MM, Haacke EM (2009) Principles, techniques, and applications of T2*-based MR imaging and its special applications. Radiographics 29:1433-1449. https://doi.org/10.1148/rg. 295095034

9. Williams A, Qian Y, Golla S, Chu CR (2012) UTE-T2* mapping detects subclinical meniscus injury after anterior cruciate ligament tear. Osteoarthritis Cartilage 20:486-494. https://doi.org/10.1016/j.joca.2012.01.009

10. Biercevicz AM, Walsh EG, Murray MM, Akelman MR, Fleming BC (2014) Improving the clinical efficiency of $\mathrm{T}^{*}$ mapping of ligament integrity. J Biomech 47:2522-2525. https://doi.org/10.1016/j.jbiomech.2014.03.037

11. Williams A, Qian Y, Bear D, Chu CR (2010) Assessing degeneration of human articular cartilage with ultra-short echo time (UTE) T2* mapping. Osteoarthritis Cartilage 18:539-546. https://doi.org/10.1016/j.joca.2010.02.001

12. Schmitz RJ, Wang H-M, Kraft RA et al (2019) Regional differences in anterior cruciate ligament imaging biomarkers: $\mathrm{T} 2$ and $\mathrm{T} 2^{*}$ values. Muscle Ligaments and Tendons J 08:238-245. https://doi.org/10.32098/mltj.02.2018.13

13. Wacker FK, Bolze X, Felsenberg D, Wolf KJ (1998) Orientation-dependent changes in MR signal intensity of articular cartilage: a manifestation of the "magic angle" effect. Skeletal Radiol 27:306-310. https://doi.org/10.1007/ s002560050387

14. Beveridge JE, Machan JT, Walsh EG et al (2018) Magnetic resonance measurements of tissue quantity and quality using $\mathrm{T} 2 *$ relaxometry predict temporal changes in the biomechanical properties of the healing ACL. J Orthop Res 36:1701-1709. https://doi.org/10.1002/jor.23830

15. Biercevicz AM, Murray MM, Walsh EG, Miranda DL, Machan JT, Fleming BC (2014) T2* MR relaxometry and ligament volume are associated with the structural properties of the healing ACL. J Orthop Res 32:492-499. https:// doi.org/10.1002/jor.22563

16. Biercevicz AM, Miranda DL, Machan JT, Murray MM, Fleming BC (2013) In situ, noninvasive, $T 2^{*}$-weighted MRI-derived parameters predict ex vivo structural properties of an anterior cruciate ligament reconstruction or bioenhanced primary repair in a porcine model. Am J Sports Med 41:560566. https://doi.org/10.1177/0363546512472978

17. Biercevicz AM, Akelman MR, Rubin LE, Walsh EG, Merck D, Fleming BC (2015) The uncertainty of predicting intact anterior cruciate ligament degeneration in terms of structural properties using $\mathrm{T}(2)\left(^{*}\right)$ relaxometry in a human cadaveric model. J Biomech 48:1188-1192. https://doi.org/10.1016/j. jbiomech.2015.02.02

18. Deoni SCL, Williams SCR, Jezzard P, Suckling J, Murphy DGM, Jones DK (2008) Standardized structural magnetic resonance imaging in multicentre studies using quantitative T1 and T2 imaging at 1.5 T. Neuroimage 40:662671. https://doi.org/10.1016/.neuroimage.2007.11.052

19. Anz AW, Edison J, Denney TS et al (2020) 3-T MRI mapping is a valid in vivo method of quantitatively evaluating the anterior cruciate ligament: rater reliability and comparison across age. Skeletal Radiol 49:443-452. https://doi. org/10.1007/s00256-019-03301-1

20. Nishii T, Kuroda K, Matsuoka Y, Sahara T, Yoshikawa H (2008) Change in knee cartilage T2 in response to mechanical loading. J Magn Reson Imaging 28:175-180. https://doi.org/10.1002/jmri.21418

21. Souza RB, Stehling C, Wyman BT et al (2010) The effects of acute loading on T1rho and T2 relaxation times of tibiofemoral articular cartilage. Osteoarthritis Cartilage 18:1557-1563. https://doi.org/10.1016/j.joca.2010.10 001

\section{Publisher's Note}

Springer Nature remains neutral with regard to jurisdictional claims in published maps and institutional affiliations. 